\title{
EXPERIMENTAL RESULTS OBTAINED WITH THE POSITRON- ANNIHILATION-RADIATION TELESCOPE OF THE TOULOUSE-ARGONNE COLLABORATION
}

J. E. Naya, P. von Ballmoos, F. Albernhe, G. Vedrenne Centre d'Etude Spatial des Rayonnements, 9, av. du Colonel-Roche, 31029 Toulouse, France

and

R. K. Smither, M. Faiz, P. B. Fernandez, T. Graber

Advanced Photon Source, Argonne National Laboratory, *

9700 S. Cass Ave., Argonne, IL 60439 USA

October 1995

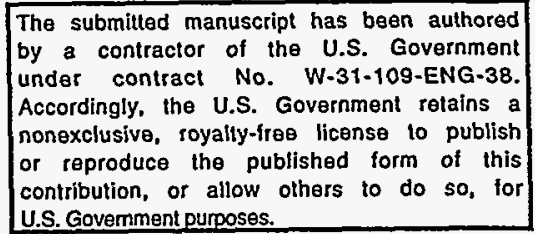

U.S. Govemment purposes.

\section{DISCLAIMER}

\begin{abstract}
This report was prepared as an account of work sponsored by an agency of the United States Government. Neither the United States Government nor any agency thereof, nor any of their employees, makes any warranty, express or implied, or assumes any legal liability or responsibility for the accuracy, completeness, or usefulness of any information, apparatus, product, or process disclosed, or represents that its use would not infringe privately owned rights. Reference herein to any specific commercial product, process, or service by trade name, trademark, manufacturer, or otherwise does not necessarily constitute or imply its endorsement, recommendation, or favoring by the United States Government or any agency thereof. The views and opinions of authors expressed herein do not necessarily state or reflect those of the United States Government or any agency thereof.
\end{abstract}

Presented at the Workshop on Imaging in High Energy Astronomy, Anacapri, Italy, September 26-30, 1995; to be published in proceedings book Imaging in High Energy Astronomy.

*This work supported by the U.S. Department of Energy, Basic Energy Sciences-Materials Sciences, under contract \#W-31-109-ENG-38. 


\title{
EXPERIMENTAL RESULTS OBTAINED WITH THE POSITRON- ANNIHULATION-RADIATION TELESCOPE OF THE TOULOUSE- ARGONNE COLLABORATION
}

\author{
J. E. NAYA, P. von BALLMOOS, F. ALBERNHE, G. VEDRENNE \\ Centre d'Eude Spatial des Rayorrements, 9, Av. du Colonenel Roche, 31029 Toulouse, France \\ and \\ R. K. SMITHER, M. FAIZ, P. B. FERNANDEZ, T. GRABER \\ Adwanced Phocon Source, Argonre National Laboratory, 9700 South Cass Avenue, Argonne, Illinois 60439 \\ USA
}

\begin{abstract}
We present laboratory measurements obtained with a ground-based prototype of a focusing positron-annihilation-radiation telescope developed by the Toulouse-Argonne collaboration. This balloon-borne telescope has been designed to collect $511-\mathrm{keV}$ photons with an extremely low instrumental background. The telescope features a Laue diffraction lens and a detector module containing a small array of germanium detectors. It will provide a combination of high spatial and energy resolution (15 arc sec and $2 \mathrm{keV}$, respectively) with a sensitivity of $-3 \times 10^{-5}$ photons $\mathrm{cm}^{-2} \mathrm{~s}^{-1}$. These features will allow us to resolve a possible narrow 511-keV line both energetically and spatially within a Galactic center "microquasar" or in other broad-class annihilators.

The ground-based prototype consists of a crystal lens holding small cubes of diffracting germanium crystals and a $3 \times 3$ germanium array that detects the concentrated beam in the focal plane. Measured performances of the instrument at different line energies (511 keV and $662 \mathrm{keV}$ ) are presented and compared with Monte-Carlo simulations. The advantages of a $3 \times 3$ Ge-detector array with respect to a standard-monoblock detector have been confirmed.

The results obtained in the laboratory have strengthened interest in a crystal-diffraction telescope, offering new perspectives for the future of experimental gamma-ray astronomy.
\end{abstract}

\section{Introduction}

Recently, the Toulouse-Argonne collaboration presented a new type of gamma-ray telescope that may begin a new stage in gamma-ray astronomy. This instrument, designed to collect $511-\mathrm{keV}$ photons with an extremely low instrumental background, consists of a Laue diffraction lens; a detector module with a $3 \times 3$ germanium array; and a balloon gondola stabilized to $5^{\prime \prime}$ pointing accuracy [1].

As a first step in the project schedule, a ground-based prototype telescope has been achieved. It consists of a diffraction lens focusing at finite distances (provided by ANL, Chicago) and a $3 \times 3$ Ge array detector (provided by the CESR, Toulouse).

In this paper, we present some of the experiments achieved and the measured performance of the system. The results obtained validate the diffraction-lens-based telescope concept and open interesting perspectives for the development of the balloon-telescope model.

\section{System test at Argonne}

The test was performed at Argonne National Laboratory in June/July 1994. It can be divided into three parts: the source, the lens module, and the detector module. 
A characteristic of the ground-based lens is the possibility of focusing a wide range of energies without retuning the lens. To accomplish this, one adjusts the distance from the source to the lens $\left(D_{s}\right)$ and the distance from the lens to the detector $\left(D_{i}\right)$ to match the corresponding Bragg diffraction angle. The relationship between these distances for small angles is:

$$
\frac{1}{D_{s}}+\frac{1}{D_{i}}=\frac{1}{F},(1)
$$

where $F$ is the focal length of the lens for a given gamma-ray energy. A general view of the setup for the two energies studied is shown in fig 1 .

\subsection{THE SOURCE}

The high-energy gamma-ray sources used in these experiments are described in table 1. They are enclosed in a large lead shield and are well collimated into a narrow cone of radiation just large enough to illuminate the lens. In this work, we present in detail the results from the $511-\mathrm{keV}$ line, which represents the energy of . interest for the proposed telescope.

\begin{tabular}{cccccc}
\hline Source & $\begin{array}{c}\text { Energy } \\
{[\mathrm{keV}]}\end{array}$ & $\begin{array}{c}\text { Activity } \\
{[\mathrm{mCu}]}\end{array}$ & $\begin{array}{c}\text { Focal } \mathbf{I} . \\
{[\mathrm{m}]}\end{array}$ & $\begin{array}{c}\mathbf{D}_{\mathbf{s}} \\
{[\mathrm{m}]}\end{array}$ & $\begin{array}{c}\mathbf{D}_{\mathbf{i}} \\
{[\mathrm{m}]}\end{array}$ \\
\hline${ }^{137} \mathrm{Cs}$ & 662 & 100 & 10.75 & 24.75 & 19.00 \\
${ }^{22 \mathrm{Na}}$ & 511 & 39 & 8.30 & 18.48 & 15.07 \\
\hline \hline
\end{tabular}

Table 1: A description of the gamma-ray sources used in this experiment is presented. Focal 1. is the focal length of the lens for the considered energy; Ds is the distance between the source and the lens; and $\mathrm{Di}$ is the distance between the lens and the projected image (where the detector is placed.)

\subsection{LENS MODULE}

The lens used in these experiments consists of 416 Ge crystals cut into small cubes of dimension $1 \mathrm{~cm} \times 1 \mathrm{~cm} \times 1 \mathrm{~cm}$. These cubes are mounted in six concentric rings on a stainless steel frame. To compenșate for the change in angle, each ring contains crystals cut so that a different set of crystalline planes is used for diffraction. The radius of each ring is designed such that each crystal in a ring focuses the same energy gamma-ray into a small focal spot simultaneously.

Due to the finite distance between the source and the lens, only a small percentage of the gamma-ray flux incident on the face of an individual crystal will have the correct Bragg angle and be diffracted. However, to optimize the diffraction efficiency of the crystals, they are divided in three parts by two wedged slots. This compensates for the change in the angle of the gamma rays as they impinge upon the front surface of the crystal.

The finite size of the source (3-mm diameter) produces a larger focal spot than would be expected for a point source $(2.0-\mathrm{cm}$ diameter instead of $1.7 \mathrm{~cm}$ for an ideal point source). For several of the experiments performed, we required a focal spot size smaller than the pixel surface, so a collimator consisting of a lead brick $(10 \mathrm{~cm}$ thick) with a cylindrical hole (8-mm diameter) was used.

The angular position of the lens axis is monitored using a laser beam reflected off a 
mirror mounted on the central axis of the lens. The 15-m-long lever arm of the laser beam allows the absolute angular position of the lens to be monitored to within a few are seconds.

\subsection{DETECTOR MODULE}

A novel gamma-ray detector consisting of a high-purity $3 \times 3$ germanium matrix housed in a single cylindrical aluminum cryostat was used for these experiments. Each of the single Ge bars is an n-type coaxial detector with dimensions of $1.5 \mathrm{~cm} \mathrm{X}$ $1.5 \mathrm{~cm} \times 4 \mathrm{~cm}$ and an internal electrode hole of 4-mm diameter. The distance between the front surface and the electrode hole is $1 \mathrm{~cm}$. Two adjacent elements are separated by $2 \mathrm{~mm}$ with an indium surface of $0.5 \mathrm{~cm}^{2}$.

Gamma-ray interactions in the array are processed and transmitted on an event-byevent basis. When an interaction is produced in one of the crystals of the array depositing an energy greater than the electronic threshold $(40 \mathrm{keV})$, a "photon detection" signal is transmitted to a PC. At this time, the computer starts a dialog with the electronics to process the event. The transmitted information consists of a series of words containing:

1) the event multiplicity (i.e., the number of crystals that have received a simultaneous energy deposit) and the identification of the triggered detector;

2) the pulse-height channel numbers, two bytes for each triggered detector.

The data are recorded in their raw form on the hard disk for a later stage of spectral analysis. The software performing the data acquisition provides facilities to both calibrate the detectors and construct spectra once the raw data file has been created. A menu allows one to easily choose any possible combination of detectors and multiplicities for elaborating the desired spectrum.

\section{Experiment results}

\subsection{LENS EFFICIENCY}

The efficiency of the lens for diffracting $511-\mathrm{keV}$ photons is given by the formula:

$$
\varepsilon_{d i f f}=\frac{A d N_{L} D_{s}^{2}}{n s N_{D}\left(D_{i}+D_{s}\right)^{2}},
$$

where $N_{L}$ is the count rate of $511-\mathrm{keV}$ events diffracted by the lens and seen by the detector; $N_{D}$ is the count rate measured by the detector when the lens is removed from the system; $D_{s}$ is the distance from the lens to the source; $D_{i}$ is the distance from the lens to the detector, $A d$ is the area of the detector, $n$ is the number of diffracting crystals in the lens; and $s$ is the average surface area of the front side of a single crystal.

In order to calculate the lens efficiency, the intensity of the diffracted beam was measured with a modified Ortec HPGe detector system, normally used at ANL, instead of the array because of its less complex geometry. The ANL detector consists of an n-type coaxial germanium detector, $6 \mathrm{~cm}$ in diameter and $6 \mathrm{~cm}$ high. The diameter of the inner hole is $1 \mathrm{~cm}$, and the distance from the front surface to the 
reentry hole has been customized to $3 \mathrm{~cm}$. This modification makes the detector more efficient at these energies than the standard model, which has a distance of 1 to $1.5 \mathrm{~cm}$ between the front face and the reentry hole.

In order to calculate the lens efficiency at $511 \mathrm{keV}$, the total counting rate of the source-lens-detector system was measured. To eliminate transmission of the nondiffracted beam through the lens and into the detector, the center of the lens was blocked. After background subtraction, we found $N_{L}=153 \mathrm{c} \mathrm{s}^{-1}$ (count rate in the peak) with the lens tuned. Next, the lens was removed from the system, and the measurement was repeated. The count rate without the lens was $N_{D}=77 \mathrm{c} \mathrm{s}^{-1}$ (in the peak).

After substitution of the parameters in formula (2), we find that the diffraction efficiency for 511-keV photons is $4.4 \%$, which corresponds to an effective diffraction surface of $17 \mathrm{~cm}^{2}$.

- The low efficiency in this case is due to the narrow intrinsic rocking curve $(\sim 2$ arc

$\because \ldots, \quad$ sec FWHM) of the crystals used in the present lens. The Na source, as seen by a narrow rocking curve, a typical crystal can only diffract photons that are emitted from a small 2 are sec strip of the source. A technique for increasing the width of a germanium crystal's rocking curve has been developed and will be incorporated in - any future lens. However, it should be noted that, if the source subtended a smaller angle, the efficiency of the present lens would increase significantly. For an astrophysical source with an angular radius on the order of 2 arc sec or less, the efficiency of present lens could be as much as $34 \%$.

\subsection{Ge ARRAY EFFICIENCY}

The efficiency calculations were carried out by comparing the measured count rates of the detector array with the count rate observed for the ANL detector. The ratio between their count rates must be equal to the ratio between their efficiencies.

\begin{tabular}{cccccc}
\hline & \multicolumn{2}{c|}{$\begin{array}{c}\text { Monte-Carlo } \\
\text { calculation }\end{array}$} & \multicolumn{3}{c}{ Measured } \\
\hline Position & $\begin{array}{c}\text { SINGLE } \\
\text { ev.eff. } \\
\text { [\%] }\end{array}$ & $\begin{array}{c}\text { MULTI } \\
\text { ev.eff. } \\
{[\%]}\end{array}$ & $\begin{array}{c}\text { SINGLE } \\
\text { ev.eff. } \\
{[\%]}\end{array}$ & $\begin{array}{c}\text { MULTI } \\
\text { ev.eff. } \\
\text { [\%] }\end{array}$ & $\begin{array}{c}\text { Backgr. } \\
\text { rejection } \\
{[\%]}\end{array}$ \\
\hline I & 12.0 & 14.9 & 12.7 & 12.4 & 79.3 \\
II & 12.9 & 18.6 & 16.4 & 16.6 & 66.3 \\
III & 12.1 & 10.8 & 12.9 & 8.3 & 83.4 \\
IV & 10.8 & 19.2 & 11.1 & 14.6 & 44.9 \\
V & 12.1 & 7.7 & 11.4 & 6.1 & 85.1 \\
\hline
\end{tabular}

Table 2: Measured and Monte-Carlo efficiencies for 511-keV photons incident on various focal spot positions on the $3 \times 3$ array. The fourth column shows the measured background rejection for each of the focal positions. 


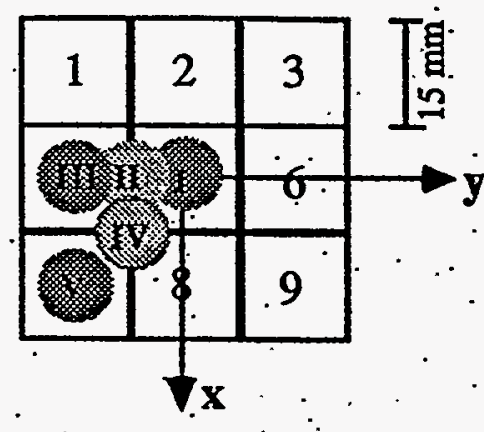

Fig. 1: Focal spot position for the table 2

The measured efficiencies for a $511-\mathrm{keV}$ gamma-ray beam incident on a $0.8-\mathrm{cm}$ diameter focal spot are shown in table 2 . We used the collimator described in the above section to limit the focal spot size to less than the pixel surface, which would be the case for the space-based telescope. The different focal spot positions referred to in the table are represented in fig. 1. We have calculated by Monte-Carlo simulations based upon CERN's GEANT [2] the photopeak efficiencies for an equivalent geometric setup. The results. are displayed in table 2 . Single and multiple events are determined by photon detection occurring in one or various pixels respectively.

\subsection{BACKGROUND REDUCTION}

The segmentation of the $3 \times 3 \mathrm{Ge}$ matrix together with the concentrated beam from the crystal lens allows application of new techniques for background reduction. The method consists of only accepting events that are compatible with the signature of a "good" 511-keV photon coming from the crystal lens. In other words, when the focal point of the lens is centered on one pixel of the array, 51i-keV photons that deposit some energy in that pixel are considered as "good" events. We can see in table 2 that the measured percentages of background rejection from an isotropic ambient background flux are associated with different positions of the focal spot. The rejection of $79.3 \%$ in the position I represents an improvement in the sensitivity by a factor of 2.2 in comparison with a standard detector of the same volume. 


\subsection{OFF AXIS SOURCE RECOGNITION}

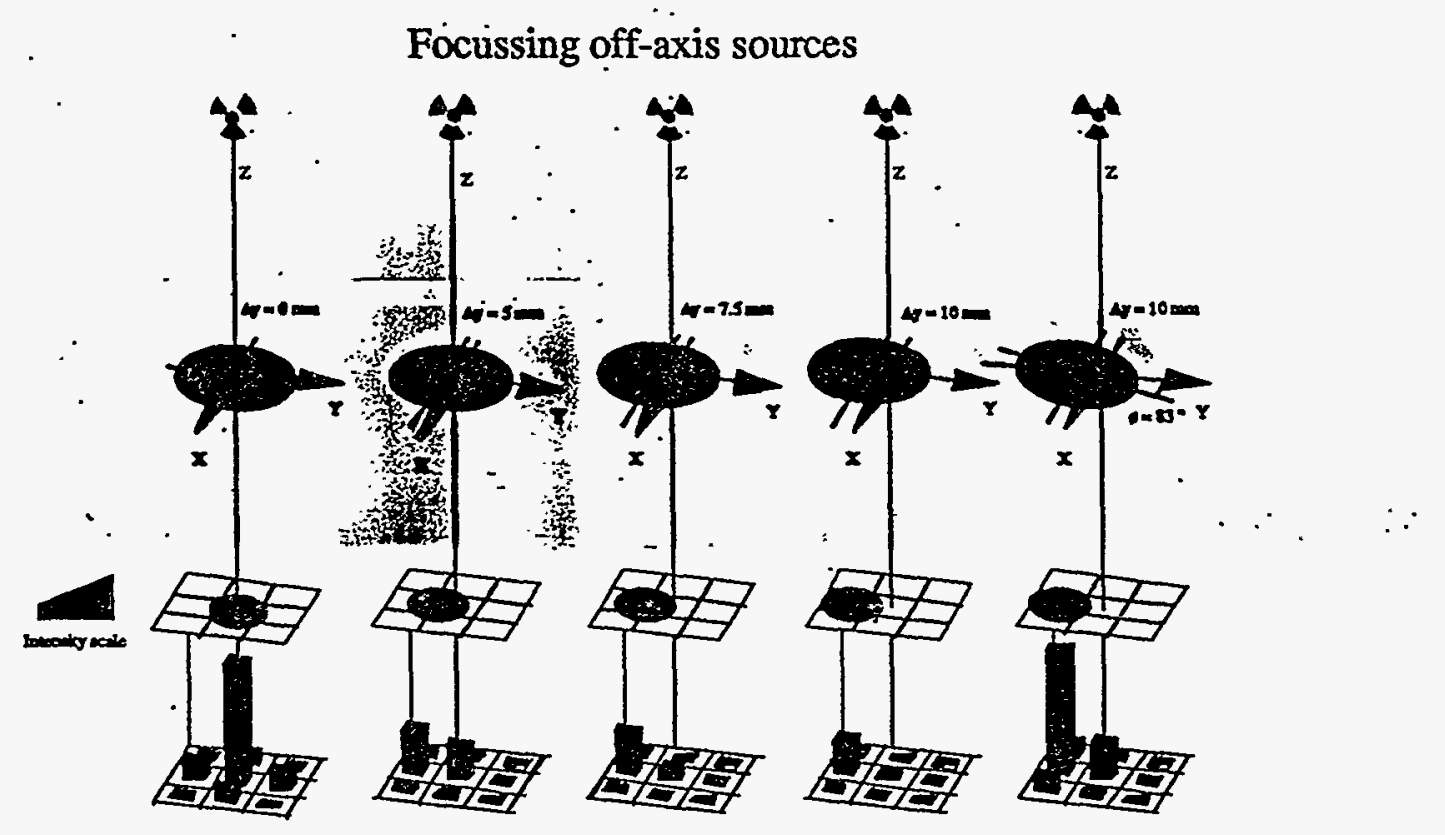

Fig. 2: The telescope response for focusing off axis sources is shown. The first drawing shows the system performance when the source, lens, and array are perfectly aligned. The following three drawings show the evolution of the focal spot position as well as the diffraction efficiency when the lens is displaced along the $Y$ axis. Finally, the last drawing shows a measurement when the lens (displaced 10 $\mathrm{mm}$ ) is tilted such that it is aligned with the source. One can see that the focal spot is on detector 4 and the initial efficiency of the system is recovered.

The germanium detector array allows us to take maximum advantage of a focused gamma-ray beam to spatially resolve the source. The focal spot can be easily localized looking at the 9 count rates. This also allows imaging of an off-axis source. Fig. 2 shows a series of measurements in which the source-lens-detector system has been intentionally misaligned. The gamma-ray energy in this experiment was 662 $\mathrm{keV}$. In the first drawing, the alignment is perfect, the focal spot being on the central pixel. The next three drawings show measurements with the lens shifted along the $Y$-axis $5 \mathrm{~mm}, 7.5 \mathrm{~mm}$, and $10 \mathrm{~mm}$, respectively. One can observe the movement of the focal spot as well as the decrease in the intensity of the focused beam due to the loses of diffraction efficiency because of the misalignment. Finally, the last drawing shows a measurement when the required tilt was applied to the lens in order to match the Bragg condition with a shift of $10 \mathrm{~mm}$. The focal spot is on detector 4, but the initial intensity of the diffracted beam has been recovered.

\section{Discussion}

The results of the tests validate the lens-telescope concept: the lens diffracts the gamma rays concentrating them into a small focal spot on the detector surface. 
Because the signal is associated with the collection surface and the background is associated with the detection volume, the large lens collection area combined with the small $\mathrm{Ge}$ array, volume makes an optimal instrument for maximizing the signalto-noise ratio.

The germanium-array-lens system also provides the following advantages:

1- The possibility of background rejection (up to $80 \%$ ) combined with a maximum detection efficiency. With the focal spot placed far from the - array borders, the efficiency increase is $25 \%$ over the nonfocused beam efficiency at $511 \mathrm{keV}$.

2- . Off-axis sounce recognition.

3- The possibility of simultaneous background source monitoring. The fact that the signal is localized to a small volume can be exploited by defining equivalent volumes within other detector pixels that are not receiving signal photons. This allows the use of these equivalent volumes to monitor the background; thus, eliminating the problem of background fluctuations during observations.

The extrapolation of these results to a balloon diffraction-lens telescope consisting of a 600-crystal lens, working at ' $511 \mathrm{keV}$, combined with the presented detector array results in an instrument with performances shown in table 3 . The calculated signalto-noise ratio of the telescope is 20 times higher than that corresponding to a spectrometer consisting exclusively of an identical Ge detector.

Such an instrument would be able to resolve, both energetically and spatially, point sources emitting narrow $511-\mathrm{keV}$ photons. Interesting candidates to observe would be the "microquasars" at the Galactic center [3], such as 1E 1740.7-258 [4] or GRS 1758-258 [5], as 'well as other objectives like Cygnus $X-1, X$-ray binaries, and pulsars.

\begin{tabular}{|c|c|}
\hline Telescope Performances & $\because \therefore$ \\
\hline 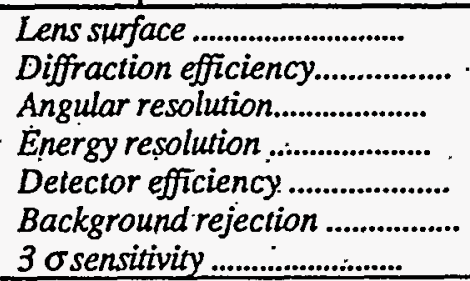 & $\begin{array}{l}600 \mathrm{crystals} 1 \mathrm{~cm}^{2} \\
25 \% \\
15 \% \\
2 \mathrm{keV} \text { at } 511 \mathrm{keV} . \\
30 \% \\
80 \% \\
310^{-5} \text { photons } \mathrm{cm}^{-2} \mathrm{~s}^{-1}\end{array}$ \\
\hline
\end{tabular}

- For a 20 hours observation mission in Alice Springs, Australia

Table 3: Performances of the proposed balloon telescope. They have been extrapolated from the ground-based telescope measurements.

$$
\therefore \quad \therefore \quad \text { Acknowledgment. }
$$

This work was partially supported by the U.S DoE Contract No. W-31-109-Eng-38.

[1] Ballimoós, P..von, et al. 1994, ApJ Sup. S., 92,663

[2] GEANT CERN Program Library Office

[3] Ramaty, R., Leventhal, M., Chan, K. W., \& Lingenfelter,'R. 1992, ApJ, 392; L63 
8

[4] Mirabel, LF., èt al. 1992, Nature, 358 (No. 6383), 215

[5] Rodriguea, L.F., et al. 1992, ApJ, 401, L15 